\title{
Overview of Intelligent Power Controller Development for Human Deep Space Exploration
}

\author{
James F. Soeder ${ }^{1}$, Timothy P. Dever ${ }^{2}$, Anne M. McNelis ${ }^{3}$, Raymond F. Beach ${ }^{4}$, and Larry M. Trase ${ }^{5}$ \\ NASA Glenn Research Center, Cleveland, $\mathrm{OH}, 44135$ \\ and \\ Ryan D. May ${ }^{6}$ \\ Vantage Partners, LLC, Cleveland, OH, 44135
}

\begin{abstract}
Intelligent or autonomous control of an entire spacecraft is a major technology that must be developed to enable NASA to meet its human exploration goals. NASA's current long term human space platform, the International Space Station, is in low earth orbit with almost continuous communication with the ground based mission control. This permits the near real-time control by the ground of all of the core systems including power. As NASA moves beyond Low Earth Orbit, the issues of communication time-lag and lack of communication bandwidth beyond geosynchronous orbit does not permit this type of operation. This paper presents the work currently ongoing at NASA to develop an architecture for an autonomous power control system as well as the effort to assemble that controller into the framework of the vehicle mission manager and other subsystem controllers to enable autonomous control of the complete spacecraft. Due to the common problems faced in both space power systems and terrestrial power system, the potential for spin-off applications of this technology for use in micro-grids located at the edge or user end of terrestrial power grids for peak power accommodation and reliability are described.
\end{abstract}

\section{Nomenclature}

AMPS $=$ Advanced Modular Power Systems

$\mathrm{BCDU}=$ Battery Charge/Discharge Unit

ISS $=$ International Space Station

MBSU = Main Bus Switching Unit

PDU $=$ Power Distribution Unit

RBI $=$ Remote Bus Isolator

RPC $=$ Remote Power Controller

\section{Introduction}

$\mathrm{N}$ ASA's present strategy for human exploration of the solar system is to "utilize incremental steps to steadily build, test, refine and qualify capabilities that lead to affordable flight elements and a deep space capability." This runs the gamut from initial exploration missions which focus primarily on visitations to the International Space Station (ISS) to "Expanding human presence into the solar system and to the surface of Mars to advance exploration..." To accomplish each of these steps, it will be necessary to develop new technologies. Many of these technologies will take many years to develop, hence, work commences now so that they will be ready when they are

\footnotetext{
${ }^{1}$ Senior Technologist for Power, Power Systems Division, 21000 Brookpark Rd./301-5, Non-Member.

${ }^{2}$ Electrical Engineer, Power Systems Division, 21000 Brookpark Rd./49-8, Non-Member.

${ }^{3}$ Aerospace Engineer, Propulsion Systems Division, 21000 Brookpark Rd./86-8, Non-Member.

${ }^{4}$ Electrical Engineer, Power Systems Division, 21000 Brookpark Rd./301-5, Non-Member.

${ }^{5}$ Electrical Engineer, Power Systems Division, 21000 Brookpark Rd./301-5, Non-Member.

${ }^{6}$ Controls Engineer, Vantage Partners, LLC, 3000 Aerospace Parkway./VPL-3, AIAA Member
} 
needed. One of the technologies that will be needed and must be addressed now is the automation of core subsystems of the spacecraft including, power, communications, thermal, avionics, propulsion, etc. The rationale for this is quite straightforward. Currently, initial human exploration missions take place aboard the ISS in low earth orbit. Communications with the station are nearly instantaneous (taking into account that they flow through the Tracking and Data Relay Satellite network in geosynchronous orbit). Consequently, most of the core systems of the ISS can either be operated from the ground or operated with a great deal of real-time ground intervention.

Contrast this with a mission to Demos and/or Phobos or a mission to the planet Mars that involves a total travel time of 6 to 9 months, and communication delays that can vary anywhere from 6 to 44 minutes ${ }^{2}$ roundtrip. Because the communication delays are so long, the mission control center on earth will no longer have the ability to assist the astronauts in real-time to diagnose and correct problems with the spacecraft. In addition, it is expected that the astronauts that will undertake such missions will not be domain experts in the respective subsystems required to operate the spacecraft. Consequently, each of the subsystems will have to have built into it a substantial amount of intelligence to permit its routine operation as well as fault diagnosis and corrective actions.

This paper describes development of an Intelligent Power Control system that can be used for autonomous power control for a deep space exploration spacecraft. This begins with a discussion of the current and future power architectures that will be supported by this type of autonomous controller. It then describes how this controller would fit into the controller architecture for the entire spacecraft. To develop the type of controller envisioned, it will be necessary to employ a detailed real-time simulation to evaluate its performance and ultimately verify its functionality. The strategy to develop this type of simulation is outlined. Finally, a discussion is presented on complementary applications such as micro-grids that are located at the edge (users) of terrestrial power grids for peak power accommodation and reliability.

\section{Power System Architecture}

Figure 1 is notional power architecture for a prospective Deep Space Vehicle. Based on studies conducted in over the last few years ${ }^{3}$, a configuration similar to this system could be used for initial deep space operations and would require an intelligent controller to manage operation.

The overall system consists of a Power Module and a Habitation Module. The Power Module can provide approximately $16 \mathrm{~kW}$ average power to the user loads. It is characterized by two independent power channels with multi-level cross strapping. Each channel has a $12 \mathrm{~kW}$ solar array and $110 \mathrm{Amp}$ hours of Lithium Ion batteries. The large arrays and batteries are needed because even though the vehicle is projected to fly in deep space there are also potential Design Reference Missions ${ }^{3}$ that require operation in environments such as low lunar orbit with an orbital eclipse/insolation time of 30 and 60 minutes respectively. Each independent power channel is $120 \mathrm{~V}$ and is compatible with SAE spec AS 5698 ${ }^{4}$. Each channel has a solar array regulator to provide conditioned power to the Main Bus Switching Unit (MBSU). From the MBSU, the power is used to charge the battery during insolation (through the battery charge/discharge unit, BCDU), or is distributed to the Power Distribution Units (PDUs), which feed the user loads. For redundancy and reliability considerations, the MBSUs can be cross-strapped and each PDU can be fed from either power channel. Finally, the MBSU can also feed bi-directional converters that can supply or draw power from the three Habitat Module docking ports. 


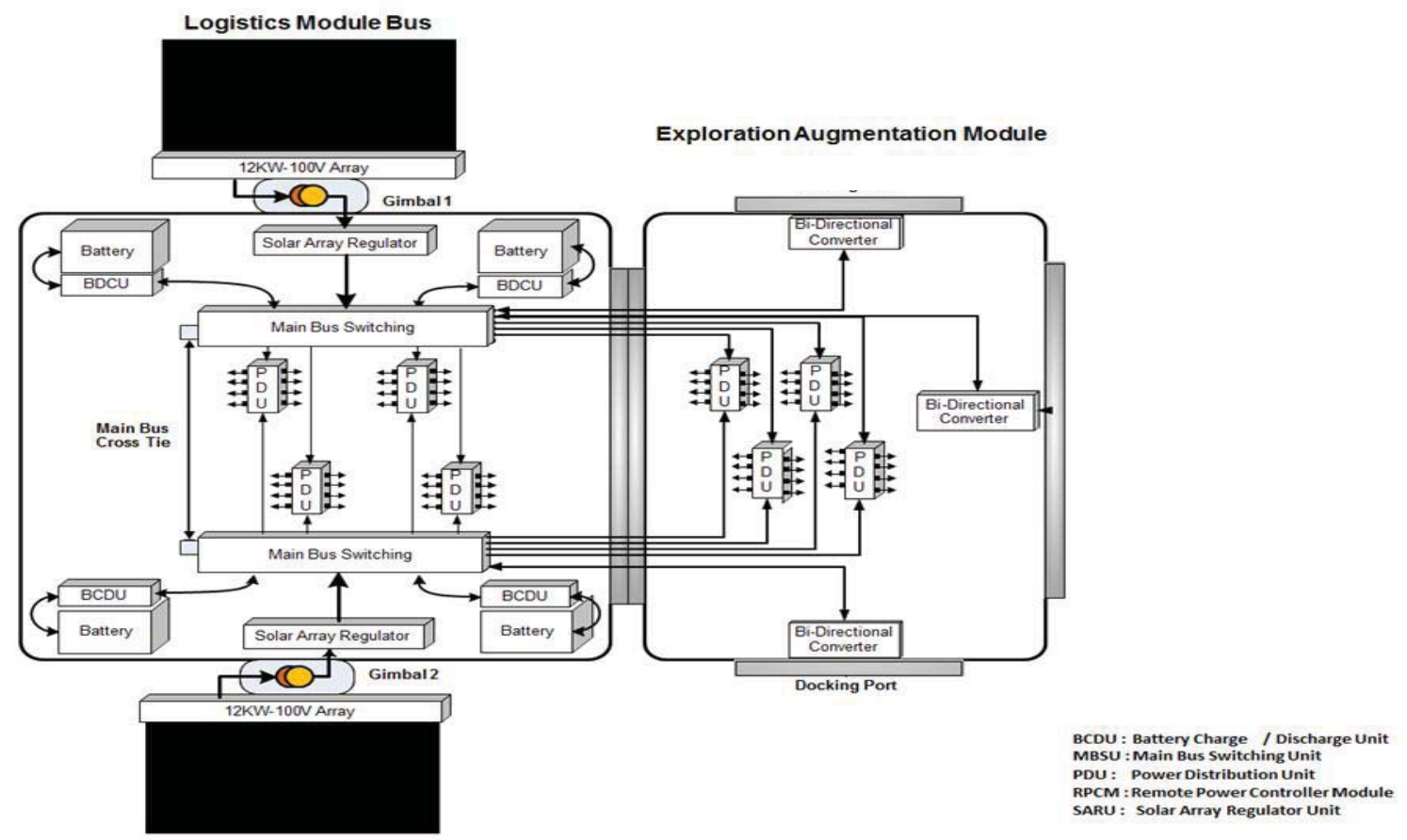

Figure 1. Notional Deep Space Vehicle Power Architecture

\section{Power Controller Architectures}

Typical controller architecture for a spacecraft is shown in Figure 2. This figure shows that each of the critical subsystems has a reactive control which performs the high speed control of the respective subsystem. For the power system this would include the trip function of the remote power controllers (solid state switches) or the regulator loops on the devices such as the solar array regulator or the battery charge/discharge control. Each of these reactive control loops report status to the on-board spacecraft controller which in turn reports status to the ground control. For flight systems such as the ISS, human ground controllers interpret ISS status and make critical decisions based on experience and mission objectives. The ground controllers may choose to alter the power profile, adjust reactive controller set points and enable or disable loads to meet the mission objectives. A key role of the ground controller is to respond to fault conditions and develop workaround plans that restore the system to fully operational status. Finally, the ground operator's role is to observe trends and anticipate the onset of problems and take corrective action to eliminate a potential fault or mitigate its impact on the system.

Once this type of information has been developed by ground control, it is then communicated to the spacecraft's controller which then relays it to the respective subsystem. Although this type of system works well in Low Earth Orbit; as human exploration pushes to Cis-Lunar space and beyond, the roundtrip communication time becomes more of an issue. Therefore, this type of system will not support the needs and constraints of human missions. 


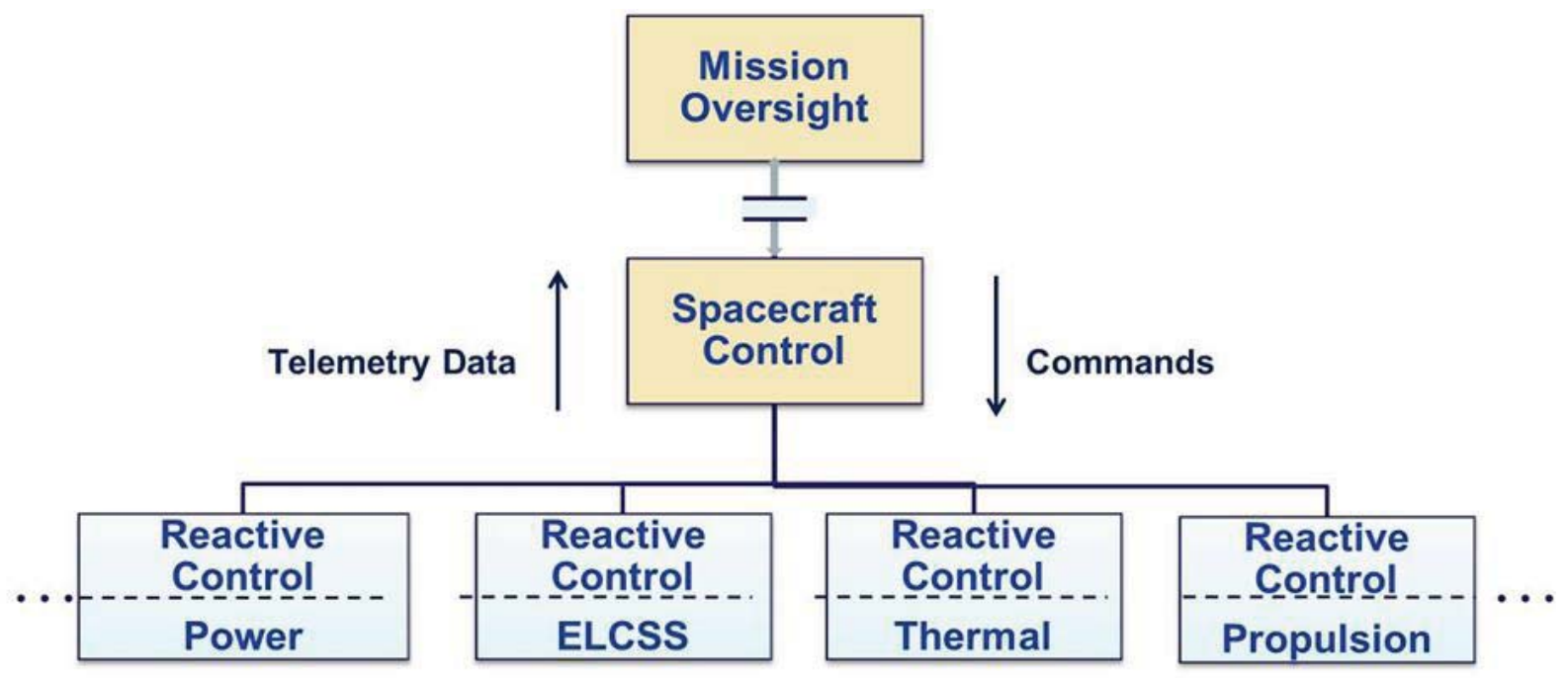

Figure 2. Simplified Spacecraft Controller Architecture

\section{Intelligent Power Control Architecture}

A potential autonomous spacecraft controller architecture is shown in Figure 3 and discussed in detail in Reference 5. In this architecture, each of the subsystems has an autonomous controller that interfaces with the respective subsystem reactive control. The autonomous controller for each subsystem manages local control issues and reports global information and issues to the spacecraft Mission Manager. For the following discussion, the autonomous controller for power will be referred to as the Intelligent Power Control. The local control problems from an Intelligent Power Control standpoint include items such as managing the energy availability, managing the power distribution network as well as detecting and reporting system failures. These functions are discussed in detail later in this section.

The spacecraft Mission Manager is responsible for managing global issues between the various subsystems as well as crew time and mission objectives. From the power system perspective, the most important global issue is load scheduling. The spacecraft's Mission Manager will provide a load schedule to the Intelligent Power Controller based on mission priorities, crew availability and inputs that it has received from the autonomous controls of the other subsystems.

Furthermore, because the Mission Manager has a global perspective of vehicle status provided by all of the subsystems, it is able to diagnose the root cause of faults that manifest themselves across more than one system. The space craft Mission Manager is then able to provide recommendations and directions to multiple subsystems to mitigate and correct the effects of power system wide faults.

Finally, the Mission Manager works in concert with ground control oversight and the crew oversight to support mission planning, fault diagnosis, etc. However, because of the long communication delays it is assumed that the on-board Mission Manager has sufficient capability to handle routine events. The mission oversight function then would become an advise and consent function as opposed the current method where all of the critical decisions are made on the ground. 


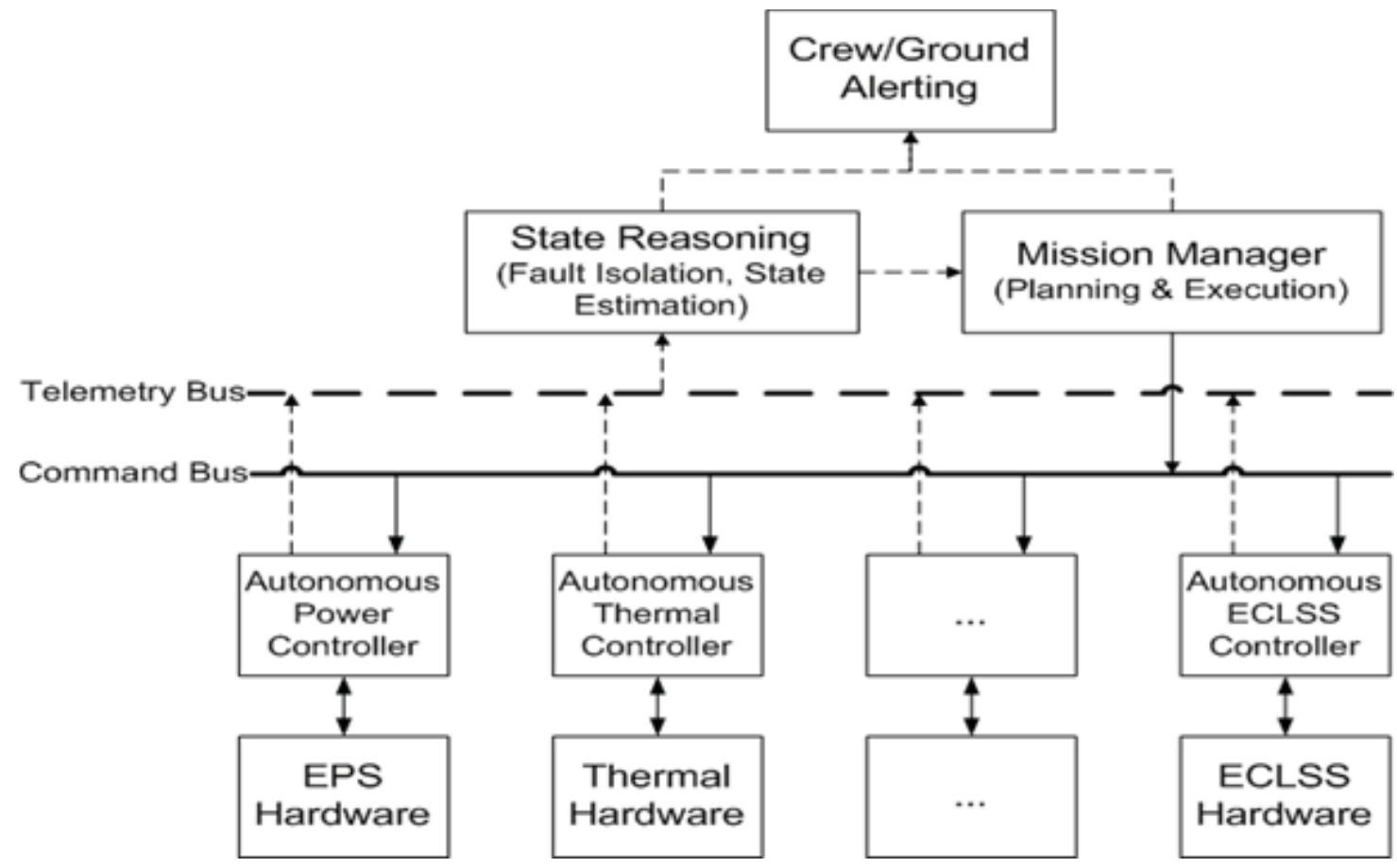

Figure 3. Intelligent Spacecraft Controller Architecture

Figure 4 is an expanded view of the Intelligent (Autonomous) Power Controller architecture in Figure 3. An early implementation of this architecture that incorporated a subset of the functions shown in Figure 3 has been developed using highly distributed software agents. This effort is discussed in detail in Reference 5. This architecture is a temporal hierarchy with the highest bandwidth elements being closest to the power components. Thus, the highest bandwidth elements are in the Reactive Control layer where each of the power system components (solar array, batteries, switchgear, power converters) has its own high bandwidth controller. The reactive controllers report critical telemetry data to the higher level control functions so that the system can operate under routine as well as fault conditions. The next layer of control receives data from the reactive layer and performs the functions of Energy Management and Power System Network Management by utilizing elements of the Power System Modelling function.

Data continues to flow up the hierarchy to the Fault Management function. This function provides for the long term monitoring of the hardware within the power system as well as for monitoring the state of the power system and the development of recovery strategies once the reactive control layer has isolated a fault and the Energy Management / Network Managements layer have identified the failure.

At the top of the power controller hierarchy is the Power Channel /Vehicle Coordination function. This acts as communications and coordination hub for the power system. The control structure shown in Figure 4 can control an entire vehicle power system or can be a cooperative element in a set of like control structures. For example, there may be two or more of the intelligent control structures, each dedicated to an individual power channel. For example, the ISS this would mean eight individual controllers - one for each channel. For a deep space habitation system there could be two controllers, one for the Habitation module and one for the Power Module.

Finally the Power Channel/Vehicle Coordination functions provides information that is needed for global decision making to the Mission Manager so that it can merged with data from other subsystems. The Mission Manager can then make the global decisions that need to take into account the entire mission needs as well as the status of all of the other spacecraft subsystems. 


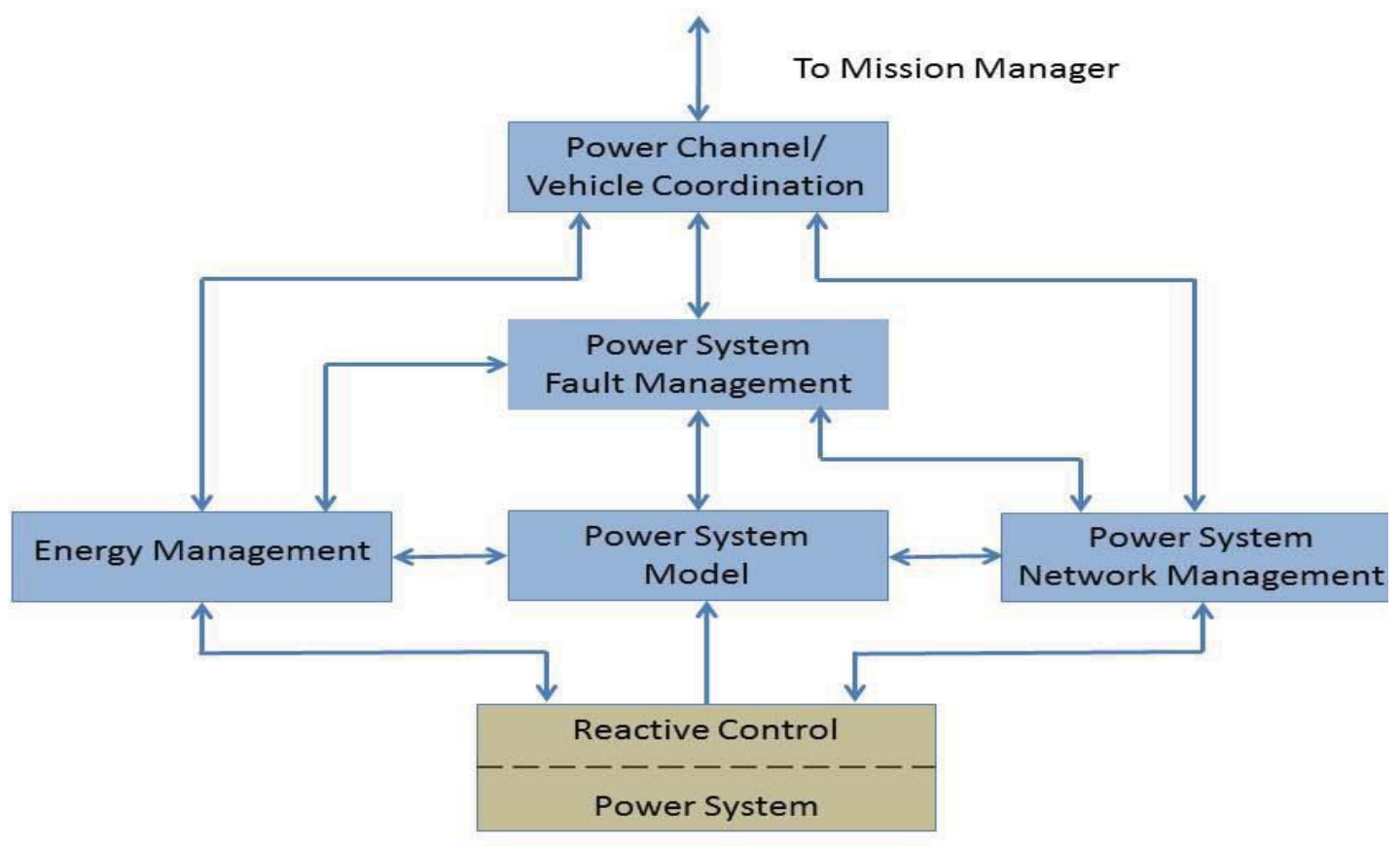

Figure 4. Intelligent Power Control Architecture

Each of the boxes shown in Figure 4 can be further decomposed into other lower level functions and actions. Shown below is a high level description and breakdown of the sub-functions for each on the boxes:

\section{A. Energy Management}

Operate and monitor the power generation and energy storage system based on external vehicle constraints as well as the system state. These functions include:

1) Maximize power availability

2) Maximize available energy storage for contingencies

3) Provide a power availability timeline

4) Provide set points for array pointing

5) Provide voltage set points for array regulation

6) Provide battery charge and discharge set points

7) Detect and report failures in the power generation and storage system

\section{B. Power Systems Network Management}

Operate and monitor the power distribution network based on load constraints as well as the overall system state. These functions include:

1) Maintain the overall power network security - ensuring that cables are not overloaded and that thermal, configuration, and safety constraints are satisfied

2) Maintain power quality - principally voltage constraints throughout the distribution system

3) Detect soft faults within the distribution system

4) Report hard faults within the distribution system

5) Send configuration information (on / off status) to the remote power controllers in the system

\section{Power System Model}

For a control of this type to operate, there needs to be high fidelity models of the power system that various control blocks can use to make decisions regarding control actions. This block provides the models and other computational capability to support those decisions and actions. These functions include: 
1) Model power generation incorporating both vehicle orbital parameters and solar array shadowing constraints

2) Model energy storage

3) Model power network (Load flow model of the distribution system)

4) Power System State Estimator

\section{Power System Fault Management}

This block manages the power system under faulted conditions and provides options for system recovery when the fault is isolated to the power system. In situations where the faults propagate to other systems it works in concert with the spacecraft Mission Manager to provide power system options to resolve the issue. These functions include:

1) Assesses and reports the state of the power system (Normal, Emergency, and Restorative) to the other autonomous (intelligent) control blocks and to the spacecraft Mission Manager. Reference 6.

2) Performs contingency analysis utilizing the power system models and sensed data

3) Recommends corrective actions based on the contingency analysis

4) Perform data trend analysis to monitor component long term health

\section{E. Power Channel/Vehicle Coordination}

This block manages the communication between the Intelligent Power Control and the Spacecraft Mission Manager. In addition, it manages communication with other power channels and/or other power systems if the overall power system is made up of two or more vehicles interconnected to form one large power system. In the case where the two vehicles are connected, the Power Channel / Vehicle Coordinators would work collaboratively together to manage the total connected power system. These functions include:

1) Communicate power system status to spacecraft Mission Manager

2) Accept commands from the spacecraft Mission Manager

3) Negotiate power channel/vehicle load profile(s) with identical entities in other channels and vehicles to maximize power availability etc. based on generation capacity, energy storage, fault condition, etc.

Each one of the control blocks consists of complex functions that need to be executed for the automated control to be operational. This is especially true of the power system fault manager that needs to analyze contingencies and provide corrective actions recommendations to the spacecraft Mission Manager.

\section{Spacecraft Mission Manager and Intelligent Power Control Interfaces}

One of the key elements necessary to the development of an Intelligent Power Controller is the definition of the external interfaces; one interface exists to the spacecraft Mission Manager and one to the reactive controls located in the power system hardware. A high-level diagram of these interfaces is shown in in Figure 5. The interface from the Reactive Controllers to the Intelligent Power Control shows all of the sensed variables that the Intelligent Power Controller needs to take control actions. These include array output voltage, battery charge / discharge voltages and currents, main bus and secondary bus currents and voltages, as well as the switchgear positions of all of the switching elements. This data, along with information from the Mission Manager is utilized by the Intelligent Power Controller to generate set points for the reactive layer such as array voltage set points, battery charge / discharge set points, RPC trip levels etc.

The interface between the spacecraft Mission Manager and the Intelligent Power Controller is more complex as it must enable the two systems to work together collaboratively. In addition, the information that must be exchanged is highly dependent on the prevailing operational constraints of the reactive layer, the vehicle configuration and operational environment. The primary purpose of this interface is to develop a plan for which system loads will be used at which time. The Mission Manager has the information necessary to develop a plan based on the state of all vehicle subsystems, crew time, and mission objectives. The Intelligent Power Controller will work with the Mission Manager to develop a load schedule that is feasible from a power perspective.

For a deep space exploration vehicle, and similar spacecraft, the Mission Manager needs to provide state vector information in addition to any mission constraints (traffic arrival schedule, viewing angle for science observation, etc.) so that any limitations on the pointing of the solar arrays can be taken into account. The Intelligent Power Controller accounts for these limitations along with state of charge of the batteries and the state of the power distribution system in order to provide the mission manager with a power availability profile for the planning period. The Mission Manager then develops an individual load profile and provides it to the Intelligent Power Controller for 
detailed analysis, plan validation and execution. Further information on this interchange can be found in Reference 7.

The Spacecraft Mission Manager also provides the Intelligent Power Controller with the Operations State of the other subsystems on-board the vehicle. This permits the Intelligent Power Controller to take into account this information when providing the Mission Manager potential corrective action recommendations in the event of a failure. Finally, the Intelligent Power Controller must provide the Mission Manager with the operating state of the EPS, the state of health of all of the power system hardware, as well as any caution and warning alarms generated by the power system. In the case where two vehicles are connected together, their respective Power Channel / Vehicle Coordinators must collaborate to provide the mission manager with unified view of the power system.

- Navigation Vectors

- Traffic / Array Pointing Constraints

- Spacecraft (other subsystems) Operations State

- Proposed load profile $\bullet$

- Array Pointing

- Array Voltage S.P.

- Battery Charge/Discharge SP.

- Switchgear Positions

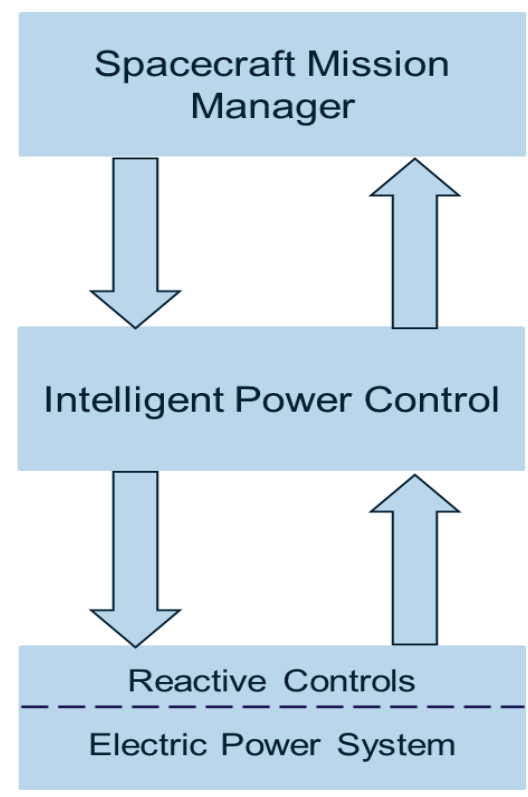

- Energy Availability

- Load Profile Evaluation

- EPS Operating State

- EPS ORU State of Health

- Caution/Warning Alarms

- Proposed Corrective Action(s)

-

- Array Voltage

- Battery Voltage

- Battery Current

- Main Bus Currents

- Main Bus Voltages

- Secondary Bus Voltage

- Secondary Bus Currents

- Switch States

Figure 5. Mission Manager Intelligent Power Control Interface

\section{Intelligent Power Controller Development and Verification}

To develop, evaluate, and verify the operation of an Intelligent Power Controller, it is necessary to run the control against a representative power system; however, it is not feasible to have all of the actual hardware necessary to properly accomplish this at the outset. In addition, it will be necessary to operate the controller in a large number of off-nominal conditions to stress the control to make sure that it is ready to safely operate the actual power hardware. Therefore, to successfully execute this task, a high-fidelity real-time simulation must be developed to exercise the control. A more detailed discussion of the overall approach can be found in Reference 8; however, an overview of the activity is outlined below.

Shown below in Figure 6 is the overall strategy for the simulation development. The left hand frame of the figure illustrates the EPS component model library which is being constructed. This consists of detailed models of Photovoltaic Cells (Solar Cells) as well as battery cells. In addition, models of power electronic elements are also being built, including isolating converters and battery charge/discharge converters. These models are state space average models to provide sufficient fidelity to evaluate the overall system response while providing real time execution speed. Finally a set of both solid state (remote power controller, RPC) and mechanical switchgear (remote bus isolator, RBI) elements have been constructed. These devices turn on and off power to various loads 
and busses and also permit the simulation of overloads and faults. This is critical if the power system simulation is to permit the evaluation of the control across its entire operating envelope.

The center frame highlights the fact that the individual components can be combined to produce larger power system units and assemblies. For example, the power converters and the PV cells can be combined to simulate a Smart Solar Array; the battery cells and the bi-directional power converter to form a Smart Battery; and the RBI and RPC elements are combined into power distribution assemblies; Main Bus Switching Units (MBSUs) for RBIs and Power Distribution Units (PDUs) for RPCs.

Finally, the third frame highlights the capability to combine the power assemblies into end-to-end power system models. By configuring the individual power assemblies, it is possible to simulate several channels of the ISS power system or alternatively the two power channels for a deep space exploration vehicle.

In order to execute the simulations shown in the $3^{\text {rd }}$ frame of Figure 6 in real time, it not only necessary to simplify the models using a such techniques as state space averaging, but it is also necessary to execute the model on multiple computer processors in parallel. Figure 7 shows the hardware architecture of the simulation environment that enables controller evaluation. The key to the system is the Distributed Heterogeneous Simulation (DHS) tool developed by PCKrause and Associates. This tool synchronizes the time-steps of simulations conducted on multiple logical processors and shares information between each of the simulations. More details about this tool and the process by which simulations can be decomposed into parallel sub problems can be found in Reference 7 .

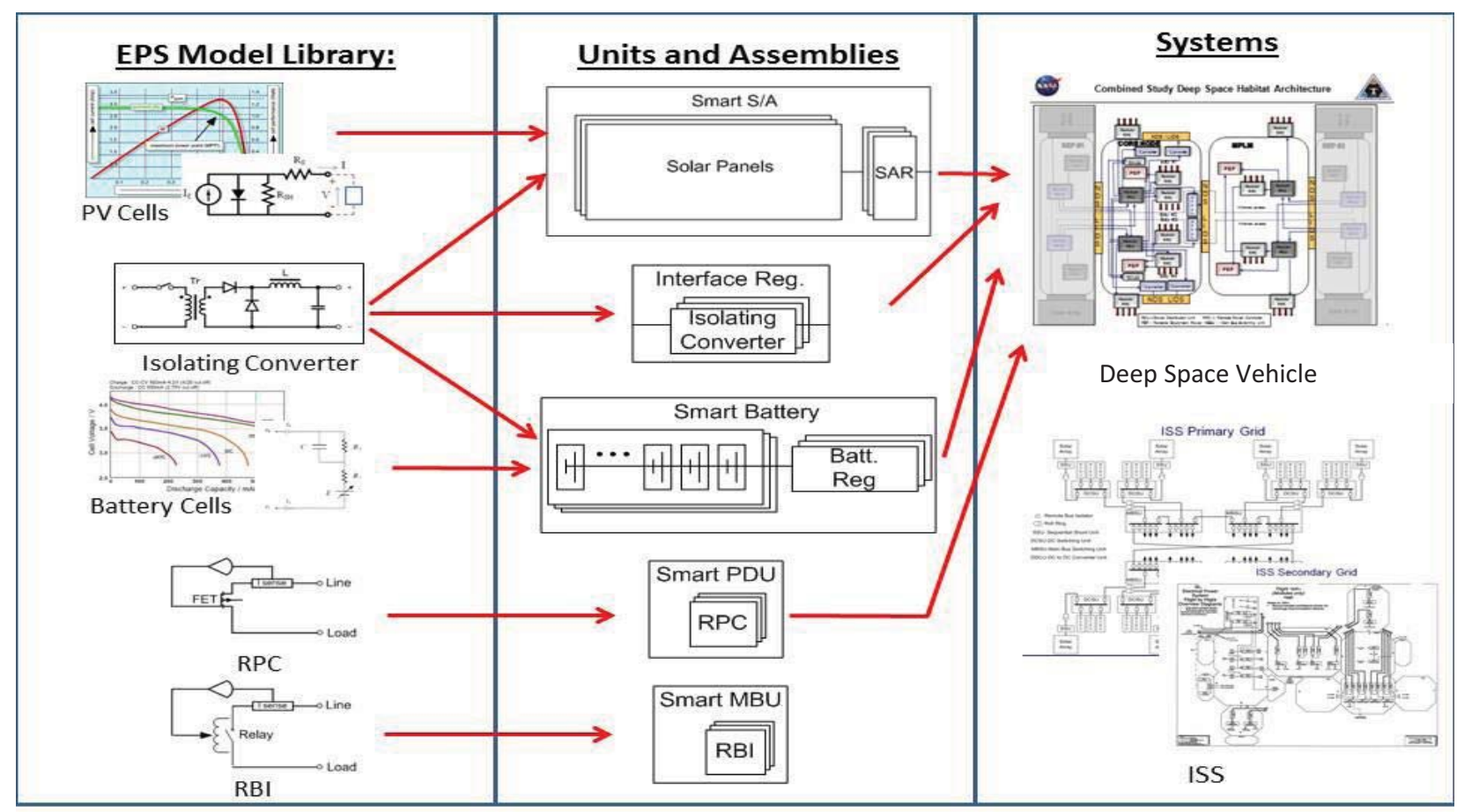

Figure 6. Simulation Development Strategy 


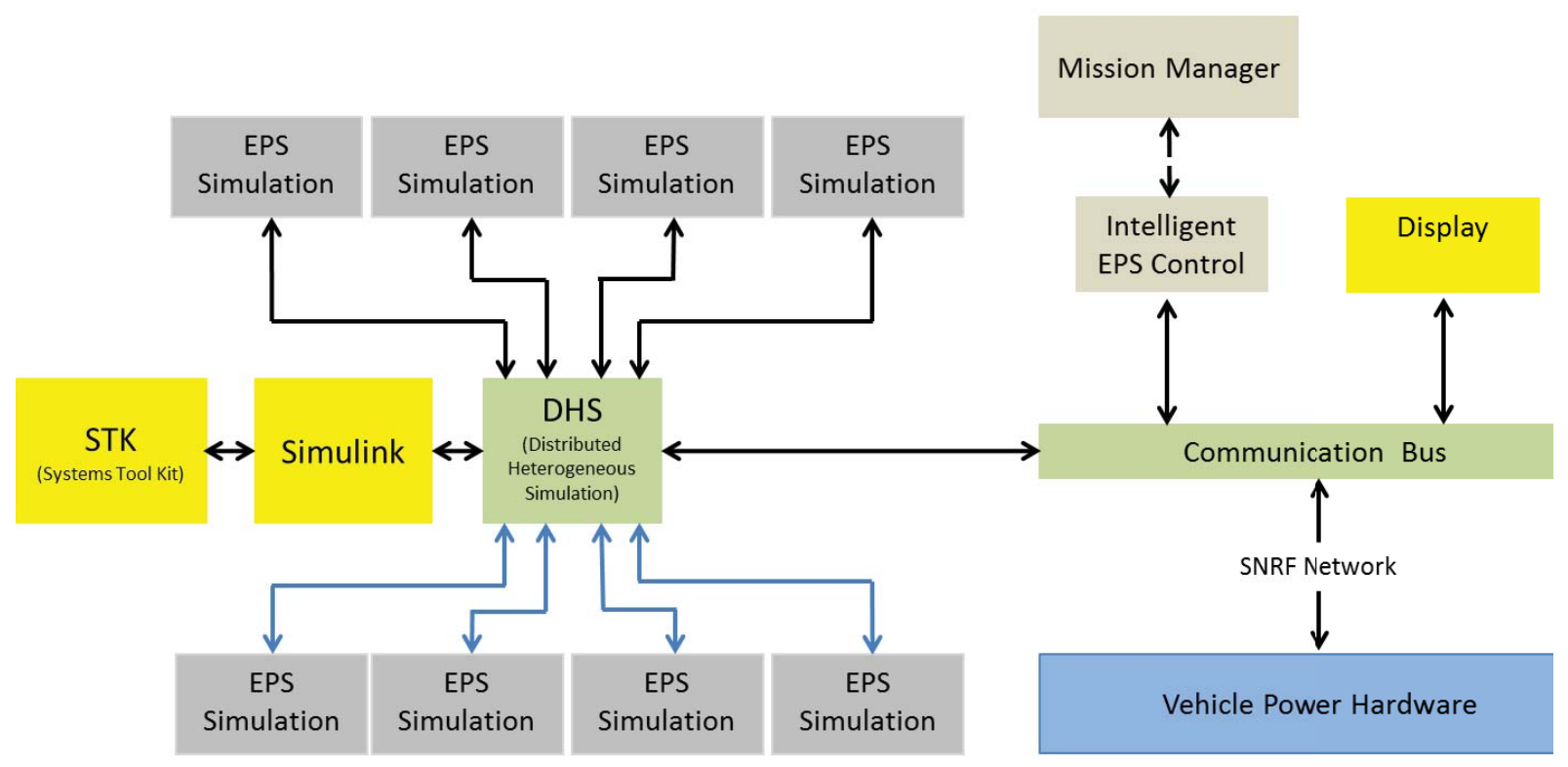

Figure 7. Intelligent Power Control Lab Architecture

The resulting simulation system enables the control system to interact with a dynamic power system simulation in real-time to investigate performance, stability, and response to faults and disturbances. Once the initial simulation work is completed, the control algorithm can be moved to hardware. The hardware implementation can then be tested against the software simulation to validate that the implementation works as expected. Finally, the software simulation can be replaced with actual power system hardware to conduct hardware validation as discussed in Reference 9. This multi-step process enables the rapid prototyping and testing of the autonomous control and enables technology development in a cost effective manner with minimal risk to hardware.

\section{Terrestrial Applications Spin-Offs}

The control architecture shown in Figure 3 is similar to the operational procedure that is used in terrestrial power grid operation centers. The Regional Transmission Organizations that are tasked with control of the transmission system create plans for system operation over many horizons (from 5 minutes to 20 years). These plans include anticipated loads based on historical trends as well as weather forecasts, anticipated generation availability, and network state. The regional grids coordinate with each other to exchange power when local deficiencies are predicted. The energy management function is cast into an Economic Dispatch problem which attempts to optimally use the available generators to meet anticipated load within the constraints of the transmission system. The plan is implemented by issuing commands to hardware throughout the system as well as commands to generators. Once operating in real time, the transmission system is constantly being monitored and a model of the system is constructed against which fault contingencies are analyzed to enable the operator to maximize system stability. All of these functions are done in a centralized manner by software with all decision making left to trained and experienced system operators.

While the spacecraft autonomous power controller is controlling a comparatively small DC electrical power system, the lessons learned and control techniques developed are almost directly applicable to the AC terrestrial system. This is particularly true in the case where there are micro grids at the edge of terrestrial power distribution networks. An example of one of these micro grids is shown in Figure 8. These micro grids can consist not only of power feeds from a utility but also local generation capability such as natural gas and geothermal generators as well as renewable sources such as wind and solar. In addition, it is expected to that these micro grids would also contain energy storage in the form of batteries, compressed air, fuel cells, etc. The management of the diverse resources under normal operating conditions as well as fault conditions poses a unique challenge. Typically, they will not be 
actively managed by either the utility or the end user directly; however, they will need sufficient management and control to insure that they provide secure reliable power under a variety of operating conditions. The control shown in Figures 3 and 4 can provide this type of capability. By first implementing this control on a spacecraft, there is a minimum of historical legacy to overcome. The experience gained from this implementation, can serve as a test bed for terrestrial development to facilitate its application and acceptance.

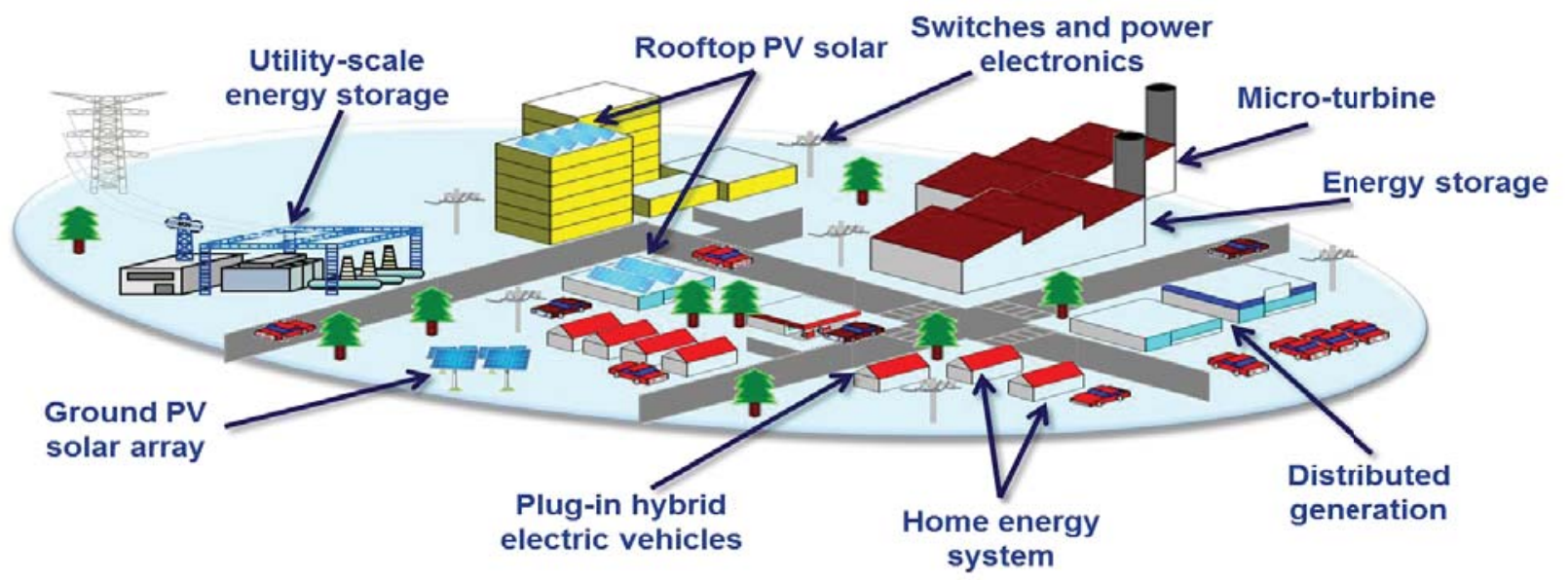

Figure 8. Terrestrial Micro Power Grid

\section{Summary}

Deep-space manned exploration requires autonomous control of all spacecraft systems to safely and effectively complete the desired missions. As a step along that path, NASA Glenn Research Center has begun development of an Intelligent Power Controller to meet this need. Collaboration with NASA Ames Research Center has highlighted how this Intelligent Power Controller can be integrated with a mission manager to begin the process of building a completely autonomous spacecraft. A path to develop and validate the Intelligent Power Controller has been identified which is comprised of initial software simulation, followed by hardware-in-the-loop testing, ultimately leading to complete hardware implementation. The lessons learned from this process can be applied to control of terrestrial power systems, particularly micro-grids.

\section{Acknowledgments}

The authors would like to thank the NASA Advanced Exploration Systems Program and Patrick George and Nancy McNelis of the Advanced Modular Power Systems Project (AMPS) for their support in this effort.

\section{References}

${ }^{1}$ NASA Strategic Plan 2014, http://www.nasa.gov/sites/default/files/files/FY2014 NASA SP 508c.pdf.

2 Jeremy, F. et.al., “Autonomous Mission Operations,” 10.1109/AERO.2013.6496927, IEEE Aerospace Conference 2013. 
${ }^{3}$ Deep Space Vehicle Power Architecture Description Document and Deep Space Habitat Test Bed Evolution, AES-AMPSRPT-002, January 15, 2013.

${ }^{4}$ SAE Space Power Standard AS5698, SAE Aerospace, February 18, 2012.

${ }^{5}$ May, R.D., Loparo, K.A., "The Use of Software Agents for Autonomous Control of a DC Space Power System," IEEE EnergyTech 2014, Cleveland, OH (submitted for publication).

${ }^{6}$ Dy Liacco, Tomas E., The Adaptive Reliability Control System, IEEE Transactions on Power Apparatus and Systems, Vol. PAS-86, No. 5, May 1967.

${ }^{7}$ May, R.D., Dever, T.P., Soeder, J.F., George, P.J. Morris, P.H., Colombano, S.P., Frank, J.D., Schwabacher, M.A., Wang, L., Lawler, D., "An Architecture to Enable Autonomous Control of Spacecraft," IECEC, Cleveland, OH (submitted for publication).

${ }^{8}$ McNelis, A.M., Beach, R.F., Soeder, J.F., McNelis, N.B., May, R.D., Dever, T.P., Trase, L., "Simulation and Control Lab Development for Power and Energy Management for NASA Manned Deep Space Missions," IECEC, Cleveland OH (submitted for publication).

${ }^{9}$ Dever, T.P., Trase, L.M., Soeder, J.F., “Application of Autonomous Spacecraft Power Control Technology to Terrestrial Microgrids," IECEC, Cleveland OH (submitted for publication). 